J. Andersen (ed.), Reports on Astronomy, Vol. XXIVA, 251-254.

(C)2000 IAU. Printed in the United States of America.

\title{
DIVISION V: VARIABLE STARS ${ }^{1}$ (ETOILES VARIABLES)
}

\section{PRESIDENT: M. Jerzykiewicz}

BOARD: L.A. Balona, E.F. Guinan, Y. Kondo, D.W. Kurtz, M. Rodonò \& J. Sahade

\author{
Commission 27: Variable Stars \\ Commission 42: Close Binary Stars
}

\section{INTRODUCTION}

This report covers the period July 1, 1996 (the closing date of the reports in the IAU Trans. 23A) through August 31, 1999. Since the reports of Commissions 27 and 42 that follow highlight the research made in the triennium, we limit ourselves here to organizational activities of the Division. The first three sections are devoted to the General Catalogue of Variable Stars (GCVS), the Information Bulletin on Variable Stars (IBVS), and the Archives of Unpublished Observations of Variable Stars, the important projects that serve us all. I am indebted to the authors, listed in the sections headings, for providing these summaries. The problems of naming and classifying variable stars, implicit in Sect. 2, are taken up in Sect. 5. The last section lists meetings sponsored by the Division.

\section{GENERAL CATALOGUE OF VARIABLE STARS (N.N. Samus)}

Two Name-lists of variable stars were published: the regular, 73rd Name-list (771 stars, IBVS 4471,1997$)$ and the special 74 th Name-list (3153 variable stars discovered by the Hipparcos mission, IBVS 4659, 1999). The GCVS names of Hipparcos new variable stars were already included in the Hipparcos catalogue (ESA 1997). In 1999, the GCVS group will submit for publication one more regular Name-list, the 75th (about 920 new variables); new special Name-lists with variable stars discovered by the CCD gravitational microlensing surveys are in preparation; the first will contain the OGLE variables. Since 1999, according to an agreement with the Bureau of Astronomical Telegrams, the GCVS team names bright galactic Novae within days after the discovery announcement, so that even early publications on a Nova contain its GCVS name.

A Supplement to the New Catalogue of Suspected Variable Stars has been made available to users by the INTERNET (announcement in IBVS 4655, 1998). It contains 11206 stars, with accurate positions and cross-identifications with more than 50 frequently used astronomical catalogues.

The GCVS group considers its first-priority task making it possible for users to find any "old" variable star, discovered before the recent large-scale surveys. Improved coordinates have been found, on the basis of identifications with positional catalogues or new astrometric measurements, for as many variables as possible in the majority of constellations of the GCVS Vol. I. In cooperation with observatories of the world, especially with the Harvard Observatory, effort is made to recover variable stars lacking finding charts.

\footnotetext{
${ }^{1}$ Division $\mathrm{V}$ has a homepage at http://www.iau.org/div5.html with links to the homepages of Commissions
} 27 and 42 . 
On the INTERNET, improved and corrected versions of the GCVS and other variablestar catalogues can be found at

http://www.sai.msu.su/groups/cluster/gcvs/gcvs/

\section{IBVS (L. Szabados, K. Oláh, A. Holl, Zs. Kövári)}

The number of published issues of the IBVS progressed from No. 4352 to 4730 . The number of subscribers for the hardcopy version is 178 .

In the past three years all issues of IBVS became available electronically, including the back issues from No. 1. The electronic IBVS has been included in the services of the Astrophysics Data System. Most of the recent users who access an issue of the electronic IBVS find it by following references through links of main journals that are also published electronically. This means that visibility of IBVS (the number of readers) has increased dramatically.

The IBVS is planned to be published on a CD-ROM with the PostScript images of issues 1-4000. Electronic links between SIMBAD and the IBVS will be established.

The Editors of the IBVS express their sincere thanks to the members of the Editorial Board: E. Budding, H.W. Duerbeck, E.F. Guinan, P. Harmanec (Chair), D.W. Kurtz, K.-C. Leung, C. Maceroni, as well as to the former member, N.N. Samus and the former Chairman, C. Sterken, who now serve as Advisors in IBVS-related matters.

\section{ARCHIVES OF UNPUBLISHED OBSERVATIONS (Edward Schmidt)}

The Archives of Unpublished Observations of Variable Stars is a depository for photometric observations which will not be published elsewhere or placed in another permanent data depository. Such details as the addresses of the archive sites, how to submit and obtain data from the archives and files can be found in the 1993 report of Commission 27.

Earlier reports of Commission 27 have listed files which were submitted to the archives. The following table lists 18 files which have been submitted to the archives since the last report. The list is complete to September 1999. This brings the total number of files currently in the archives to 308 . All of the files listed here are available in electronic form.

\begin{tabular}{lll} 
File & Contributor(s) & Star(s) \\
\hline 279 & Poretti & $\beta$ Cas \\
306 & Poretti & AZ Cmi, BF Phe, SAO 4710 \\
315 & Morris \& Naftilan & RZ Tau \\
316 & Ceruti & AG Phe \\
317 & Heckert & E 1919+0427 \\
318 & Krisciunas & F stars in M34 \\
320 & Rodriguez & AN Lyr \\
321 & Rodriguez & V1719 Cyg \\
322 & Heckert & WY Cnc \\
323 & Rodriguez & AN Lyn \\
326 & Linnell & MR Cyg \\
332 & Rodriguez & 20 Cvn \\
333 & Schmidt & V719 Her \\
334 & Rodriguez & 28 And \\
335 & Rodriguez & BS Aqr \\
336 & Rodriguez & AB Cas \\
337 & Rodriguez & HD 163151 \\
338 & Snyder & UV Leo \\
339 & Fernie & $\delta$ CrB \\
\hline
\end{tabular}




\section{NAMING AND CLASSIFYING VARIABLE STARS}

As it is clear from Sect. 2, Kolya Samus and his co-workers are making heroic efforts to keep track of the hundred-thousands variables discovered as by-product of the CCD microlensing surveys. Without help, especially from the discoverers themselves, they cannot possibly succeed. The discovery papers should include instructions how to locate the new variables on the sky, so that other workers will have a chance of verifying the original findings. Without this it may prove easier to re-discover a variable than find it among those already known.

Another problem is the availability of photometric (and other) data. The IAU should encourage (and, perhaps, assist financially) setting up and maintaining electronic data bases. In addition to current research, these data should be available to interested workers for decades to come, so that problems such as long-term variability of mean magnitudes, periods and amplitudes could be addressed. The examples to follow are the Archives of Unpublished Observations (Sect. 4) and the practice of the OGLE collaboration (e.g., Udalski et al. 1994).

The GCVS system of classification of variable stars is a useful first approximation, although the catalogue's notation is seldom used in the literature (with the notable exception of the Hipparcos Catalogue). Instead, experts in particular fields of stellar variability develop their own terminology. One reason may be that the GCVS system is based primarily on the light-curve morphology, so that stars which show radial-velocity or line-profile variations but were not found to be variable in brightness are left out. The question is whether we should try to improve the situation. If enough people felt the answer is yes, a WG on Variable Stars Terminology could be organized.

\section{MEETINGS}

Division V sponsored, co-sponsored or coordinated the following Symposia, Colloquia, and Joint Discussions (in chronological order; editors of proceedings are listed in parentheses; if the proceedings were published only in Highlights of Astronomy, the parentheses contain the title and the chairperson). JD8 (Guinan \& Koch 1999), JD12 (Electronic Publishing: Now and the Future, A.G. Hearn), JD14 (The First Results of Hipparcos and Tycho, C. Turon), JD24 (Takeuti \& Sasselov 1998), C175 (Smith, Henrichs \& Fabregat 2000) and C176 (Szabados \& Kurtz 2000). In addition, Commission 27 supported S181 (Provost \& Schmider 1997), S185 (Deubner, Christensen-Dalsgaard \& Kurtz 1998) and S191 (Le Bertre, Lèbre \& Waelkens 1999), while Commission 42 co-supported S188 (Koyama, Kitamoto \& Itoh 1998).

As to the two most recent meetings, C175 and C176, the latter is covered in the report of Commission 27 (this volume), while a summary of the former can be found at the URL: http://www.bestars.ua.es/be99.

Acknowledgments. The WWW homepages of Commissions 27 and 42 are maintained by Andras Holl of the Konkoly Observatory. We are indebted to him for this important service to the community.

M. Jerzykiewicz

President of the Division

\section{References}

Deubner, F.-L., Christensen-Dalsgaard, J., Kurtz, D. W., eds., 1998, IAU Symposium 185, New Eyes to See Inside the Sun and Stars (Dordrecht: Kluwer Academic Publishers)

ESA, 1997, The Hipparcos and Tycho Catalogues, ESA SP-1200

Guinan, E.F., Koch, R., eds., 1999, IAU XXIII GA JD8, New Astr., Stellar Evolution on Human Time Scales (Amsterdam: Elsevier Science B.V.) 
Koyama, K., Kitamoto, S., Itoh, M., eds., 1998, IAU Symposium 188, The Hot Universe (Dordrecht: Kluwer Academic Publishers)

Le Bertre, T., Lèbre, A., Waelkens, C., eds., 1999, IAU Symposium 191, Asymptotic Giant Branch Stars (San Francisco: ASP)

Provost, J., Schmider, F.-X, eds., 1997, IAU Symposium 181, Sounding Solar and Stellar Interiors (Dordrecht: Kluwer Academic Publishers)

Smith, M., Henrichs, H., Fabregat, J., eds., 2000, IAU Colloquium 175, ASP Conf. Ser., The Be Phenomenon in Early-Type Stars (San Francisco: ASP)

Szabados, L., Kurtz, D. W., eds., 2000, IAU Colloquium 176, ASP Conf. Ser., The Impact of Large-Scale Surveys on Pulsating Star Research (San Francisco: ASP)

Takeuti, M., Sasselov, D.D., eds., 1998, IAU XXIII GA JD24, Pulsating Stars: Recent Developments in Theory and Observation (Tokyo: Universal Academy Press)

Udalski, A., Kubiak, M., Szymański, M. et al., 1994, Acta Astron., 44, 317 